\title{
Editorial
}

\section{Nathalie Touze-Foltz}

President, French Chapter of the International Geosynthetics Society; Head, Irstea regional centres, Antony and Nogent, France

The specific hydraulic properties of bentonite such as hydration, swelling, water absorption, or mechanical properties such as viscosity, yield stress and thixotropy make it a valuable material in the form of mineral powder. Many industrial applications of bentonite exist, from pharmacology and the agrifood sector where bentonite is specifically purified and controlled for its consumption (natural filters, cremes, gastric protectants), to civil engineering where the ground raw material, possibly activated, is used in large quantities in drilling fluids, cut-off walls and barriers, for example.

One of the civil engineering applications that are not covered in this issue of Environmental Geotechnics is the use of bentonite inside a geosynthetic called geosynthetic clay liner (GCL) or clay geosynthetic barrier (CGB). Geosynthetics is the generic name of a large family of manufactured products, at least one of whose components is made from a synthetic or natural polymer, in the form of a sheet, a strip or a three-dimensional structure, used in contact with soil and/or other materials in geotechnical and civil engineering applications. GCLs are defined as an assembled structure of geosynthetic materials and a low hydraulic conductivity earth material (clay that often is a bentonite) in the form of a manufactured sheet that is used in civil engineering applications. The function of GCLs, thanks to the bentonite layer they contain, once confined and hydrated, is to ensure lining, thus prevent fluid and contaminant transport.

In recent years, there have been many advances in the understanding of issues related to the use of GCLs: bentonite hydration and swell in connection with unsaturated phenomena; influence of the hydration fluid on swell and resulting hydraulic performance of the GCL; cation exchange, with or without interaction with freeze/thaw and hydration desiccation processes; polymer-enhanced bentonites - all in an attempt to quantify the best manufacturing, design and installation to ensure long-term hydraulic performance of these materials. Mechanical aspects have also been in the loop of focus; dealing with confinement of GCLs and stability issues is especially meaningful when multiple and complex interfaces are involved, for example, in landfill slopes or covers. As a result, as time goes, the knowledge in the behaviour of GCL properties and performance increases and their behaviour in the long term is increasingly better controlled. This knowledge accumulated over time has been spread through education in the various spheres involved, from decision to installation. Consequently there has been a significant increase in geoenvironmental applications of those materials. GCLs are now used at more and more landfills and mining sites all around the world, in an attempt to improve the environmental protection around those sites, controlling fluid migration.
Bentonites and environmental protection are at the heart of this issue of Environmental Geotechnics. The four papers included in this issue bring significant knowledge that should serve as a basis to improve future uses of bentonite in environmental protection.

The capability of bentonite to serve as a barrier to fluids is intimately linked to the uptake of moisture by the bentonite. Consequently the hydration process of the bentonite is an important topic as previously mentioned. The paper presented by Katti et al. (2017) focuses on the modelling of the hydration and swelling of montmorillonite at the molecular scale, in the presence of organic molecules present in landfill leachate. It provides insight into molecular mechanisms that lead to macro-scale behaviour of swelling clays. Takayama et al. (2017) discuss in a different paper the modelling of the unsaturated hydromechanical behaviour of bentonite during saturation for storage in nuclear waste disposal facilities.

In nuclear waste disposal facilities, like in landfills or in cut-off walls (which are discussed in the paper by Alzayani et al. (2017)), the bentonite function is to ensure lining. One could conclude too hastily that only hydraulic properties matter. However, understanding the hydromechanical behaviour of bentonite is of primary importance to understand its limits and use this material in the best possible conditions to ensure durability and protection of the surrounding environment on the long term, thus sustainability. The elaboration of the models presented in this issue by Alzayani et al. (2017) and Takayama et al. (2017) at the forefront of research can certainly be beneficial to move forward in the field of GCLs hydromechanical behaviour as far as the bentonite component in these materials is concerned.

Finally, Sarkar et al. (2017) present an approach to prepare bentonite nanoparticles that can be used to reduce the hydraulic conductivity in barrier materials or for the removal of pollutants.

It is interesting to see that as the performance in terms of environmental protection is concerned, in this issue of Environmental Geotechnics the focus is on similar concerns as in the field of GCLs

- hydration/saturation to guarantee the best possible hydraulic performance in the long term

- unsaturated hydromechanical behaviour, as stresses will affect the liner performance in relation with the saturation state of the bentonite

- interaction with contaminants that can influence the hydraulic performance or the bentonite 
- attenuation or pollution remediation through sorption mechanisms that will slow down their transport.

Irstea, the French National Institute for Research in Agriculture and Environment, has been involved in the study of the performance of geosynthetics in environmental applications for more than 25 years. My hope is that after reading this Editorial, readers of Environmental Geotechnics that have been interested in this issue will be interested in reading more about geosynthetics and environmental protection. I do also hope that researchers in the field of geosynthetics will use the interesting matter in this issue to enrich their knowledge in the field of bentonite behaviour.

The significant matter presented here should lead to enhancement of models, to keep on predicting in an improved manner the behaviour of GCLs. I trust that more cooperation between geoengineering communities is an achievable goal, with the final aim to bring better protection to our environment. I warmly thank the Editorial Board for giving me, as a member of the International Geosynthetics Society, an opportunity to contribute to this issue.

\section{REFERENCES}

Alzayani NJ, Royal ACD, Ghataora GS and Jefferson I (2017) Cementbentonite in comparison with other cemented materials. Environmental Geotechnics 4(5): 353-372, http://dx.doi.org/10.1680/jenge.14.00050.

Katti DR, Patwary ZR and Katti KS (2017) Modelling clay-fluid interactions in montmorillonite clays. Environmental Geotechnics 4(5): 322-338, http://dx.doi.org/10.1680/jenge.14.00027.

Sarkar G, Dey A and Siddiqua S (2017) Preparation of Wyoming bentonite nanoparticles. Environmental Geotechnics 4(5): 373-381, http:// dx.doi.org/10.1680/jenge.15.00001.

Takayama Y, lizuka A and Kawai K (2017) Simulation of a saturation process in a transuranium disposal facility. Environmental Geotechnics 4(5): 339-352, http://dx.doi.org/10.1680/jenge.14.00041. 